\title{
Guideline Discordance and Patient Cost Responsibility in Medicare Beneficiaries With Metastatic Breast Cancer
}

\author{
Courtney P. Williams, MPHª; Andres Azuero, PhD, MBA ${ }^{\text {b; }}$ Kelly M. Kenzik, PhD, MSc,d; Maria Pisu, PhD d,e;
} Ryan D. Nipp, MD, MPH; Smita Bhatia, MD, MPHc; and Gabrielle B. Rocque, MD, MSPHa,d

\begin{abstract}
Background: Treatment for metastatic breast cancer (MBC) that is not concordant with the NCCN Guidelines for Breast Cancer has been associated with higher healthcare utilization and payer costs. However, a significant knowledge gap exists regarding the impact of guideline-discordant care on patient cost responsibility. This study examined this question among patients with $\mathrm{MBC}$ in the year postdiagnosis. Methods: This retrospective cohort study used data from the SEER-Medicare linked database from 2000 through 2013. Guideline discordance, defined by year-specific NCCN Guidelines, was assessed for first-line antineoplastic treatment and grouped into discrete categories. Patient cost responsibility (deductibles, coinsurance, copayments) in women with MBC were summed for all medical care received in the year postdiagnosis. The difference in patient cost responsibility by guideline discordance status was estimated using linear mixed-effect models. Results: Of 3,709 patients with $\mathrm{MBC}$ surviving at least 1 year postdiagnosis, $17.6 \%(n=651)$ received guideline-discordant treatment. Median cost responsibility in the year postdiagnosis for patients receiving guideline-discordant treatment was \$7,421 (interquartile range [IQR], \$4,359-\$12,983) versus $\$ 5,171$ (IQR, $\$ 3,006-\$ 8,483$ ) for those receiving guidelineconcordant care. In adjusted models, guideline-discordant treatment was significantly associated with $\$ 1,841$ higher patient costs in the first year from index diagnosis date $(95 \% \mathrm{Cl}, \$ 1,280-\$ 2,401)$ compared with guideline-concordant care. Patient cost responsibility differed by category of guideline discordance, with those receiving nonapproved bevacizumab having the highest cost responsibility $(\beta=\$ 3,330 ; 95 \% \mathrm{Cl}, \$ 1,711-\$ 4,948)$. Conclusions: Deviations from current treatment guidelines may have implications on patient healthcare cost responsibility. Additional research is needed to fully understand the mechanisms underlying how guideline deviation leads to greater costs for patients with MBC.
\end{abstract}

J Natl Compr Canc Netw 2019;17(10):1221-1228 doi: $10.6004 /$ jnccn.2019.7316

aDivision of Hematology and Oncology, b ${ }^{b}$ chool of Nursing, Institute for Cancer Outcomes and Survivorship, ${ }^{\mathrm{d}} \mathrm{O}$ 'Neal Comprehensive Cancer Center at $\mathrm{UAB}$, and ${ }^{\mathrm{E}}$ Division of Preventative Medicine, University of Alabama at Birmingham, Birmingham, Alabama; and fDivision of Hematology and

Oncology, Massachusetts General Hospital Cancer Center and Harvard Medical School, Boston, Massachusetts.

\section{Background}

The complexity of cancer care continues to increase, and thus oncologists rely heavily on NCCN treatment guidelines for informed decision support at the point of care. NCCN Clinical Practice Guidelines in Oncology (NCCN Guidelines) include evidence-based, physician-recommended treatments. Concordance with NCCN Guidelines for Breast Cancer is increasingly recognized as a marker of high-quality cancer care. ${ }^{1,2}$ Specifically in metastatic breast cancer (MBC), $>45$ guideline-based treatment options exist, providing room for treatment personalization. ${ }^{3}$ According to previous research, approximately one-fifth of Medicare beneficiaries with MBC have received treatment discordant with NCCN Guidelines. ${ }^{4,5}$ These patients had almost \$2,000 USD higher monthly Medicare spending compared with those who received guideline-concordant care, yet no survival difference was seen between the groups. Although these studies found higher Medicare spending associated with NCCN Guideline discordance, a knowledge gap remains about how guideline discordance may affect patient-specific cost responsibility.

Increasingly, oncologists have begun to recognize the importance of patient-specific cost responsibilities, defined as the healthcare service payment owed by the patient and not by a third party, including deductibles, coinsurance, and copayments. These responsibilities have steadily increased for patients with cancer due to expensive new treatments, frequent clinic visits, and increasing survival. Recently, the oncology community has begun to consider the patient-level financial burden and distress caused by cancer treatment a new adverse effect, termed "financial toxicity." 6 Financial toxicity has been linked to negative patient outcomes, such as medication nonadherence, high symptom burden, and poor quality of life. ${ }^{7,8}$ Older adults (aged $\geq 65$ years) are particularly vulnerable to financial toxicity, because they often live on a fixed income and therefore could be financially and psychologically strained when faced with high cancerrelated care costs. Given that guideline-discordant treatment resulted in higher Medicare spending according to previous studies, it may also impact the costs for which 
patients are responsible, and thus potentially increase financial toxicity in older patients with MBC. No prior work has differentiated the patient cost responsibility between those receiving guideline-discordant versus -concordant treatments. Therefore, we sought to determine the patientlevel impact of NCCN Guideline-discordant treatment on patient cost responsibility in the year after index diagnosis for Medicare beneficiaries with MBC.

\section{Patients and Methods}

\section{Study Design and Sample}

This retrospective cohort study used data from 2000 through 2013 in the SEER-Medicare linked database to examine the association between guideline discordance and patient cost responsibility in women with MBC. ${ }^{9}$ Women with de novo (metastatic at diagnosis) MBC diagnosed in 2007 through 2013 or with stage I-III breast cancer diagnosed in 2000 through 2013 with subsequent secondary distant metastases in 2007 through 2013 who survived 1 year after index diagnosis were included in this study (Figure 1). ${ }^{4,5}$ Metastatic disease was identified based on ICD-9 claims on 2 separate dates for secondary cancer (197.XX-198.XX), excluding breast (198.81, 198.82, 198.2) and lymph nodes (196.XX). ${ }^{10,11}$ The index diagnosis date was defined as the de novo MBC diagnosis or the first claim date with ICD-9 codes for secondary cancer in patients with a primary early-stage breast cancer. Exclusion criteria included no anticancer treatment (chemotherapy, hormone therapy, targeted therapy), other nonbreast primary cancers, unknown hormone receptor status, or incomplete Medicare coverage (Parts A, B, D) in the year after index diagnosis. This study was approved by the University of Alabama at Birmingham Institutional Review Board. No informed consent was required because the analysis used deidentified data from the SEER-Medicare database.

\section{Outcome Measure}

The outcome of interest, patient cost responsibility, was defined as payment to providers for which Medicare beneficiaries were responsible through deductibles, coinsurance, and copayments, in the year after index diagnosis date. ${ }^{12}$ Overall and healthcare service-stratified costs to beneficiaries were summed from inpatient (Medicare Provider Analysis and Review), outpatient (Outpatient Standard Analytical Files), physician visit/ carrier (National Claims History), durable medical equipment (DME), and Medicare Part D claims. Notably, data for Medicare Part B drug spending, which includes almost all infusion chemotherapy drugs and some oral chemotherapy drugs given at a doctor's office, freestanding clinic, or hospital outpatient setting, was found in the outpatient, physician visit/carrier, and DME claim files.

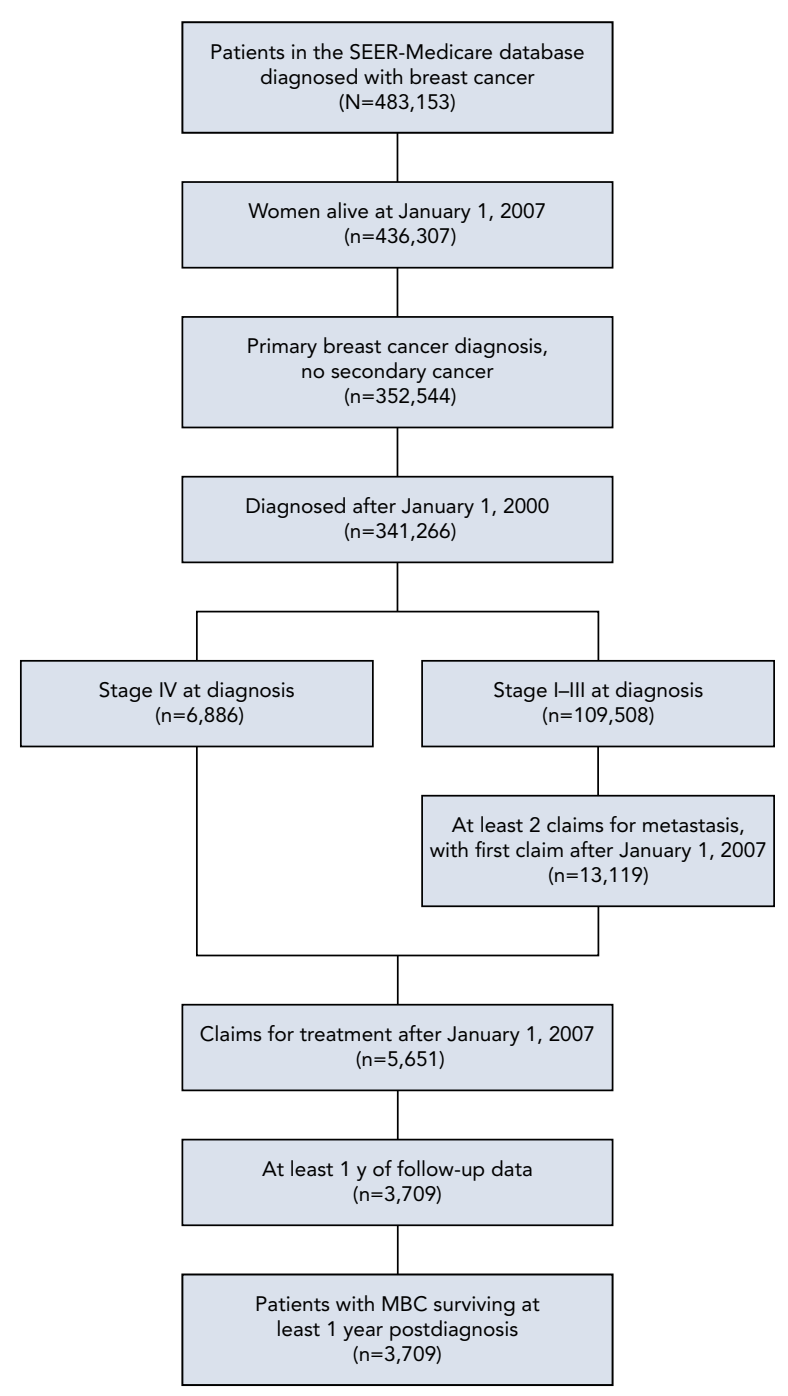

Figure 1. Exclusion cascade.

Abbreviation: MBC, metastatic breast cancer.

\section{Guideline Discordance}

NCCN Guideline discordance was assessed using claims for initial antineoplastic treatment (hormonal medication, chemotherapy, or HER2-targeted therapy) after index diagnosis date. Details regarding the categorization of guideline discordance in this population were previously published. ${ }^{4,5}$ In brief, treatment regimens containing single drugs or combination therapy (same-day billing for $\geq 2$ drugs) were identified using National Drug Codes, the Healthcare Common Procedure Coding System, and generic drug names from outpatient, physician visit/ carrier, DME, or Medicare Part D claims. Regimens were matched to the version of NCCN Guidelines for Breast Cancer available at the exact treatment date. Regimens considered guideline-concordant changed over time, because the NCCN Guidelines are updated at least annually or as needed based on changes in clinical 
evidence for existing drugs or new drug approvals. However, minor changes were observed during the time frame of our study. Guideline discordance was defined as regimens received but not listed in the published NCCN Guidelines at time of treatment. Patients with unknown HER2 status were considered concordant if they received a HER2-positive or -negative regimen. Treatment regimens categorized as guideline-discordant were subsequently reviewed and grouped into discrete categories based on the reason for discordance, including (1) therapy mismatched with hormone receptor (HR) or HER2 status, (2) HER2-targeted therapy without chemotherapy, (3) nonapproved bevacizumab use, (4) adjuvant regimens received in the metastatic setting, and (5) miscellaneous reasons for guideline discordance, which included nonapproved agents or regimens usually received in cancers other than $\mathrm{MBC}$, trastuzumab in nonapproved combinations, and approved agents or regimens received in nonapproved years. ${ }^{4,5}$

\section{Covariates}

Patient demographic and clinical characteristics were compared and used for model adjustment, including age at index diagnosis, race (white, black, other) type of MBC (de novo vs treated, secondary metastatic disease), HR status (positive vs negative vs unknown estrogen receptor [ER]/progesterone receptor status), HER2 status (positive vs negative vs unknown), NCI comorbidity index score, Medicare/Medicaid dual-eligibility status (never eligible vs eligible), and census track education level (percentage of patients with less than high school education vs high school education or greater). NCI comorbidity index scores were based on claims for comorbid conditions in the year before the index diagnosis date and classified based on the Klabunde modification for comorbidities. ${ }^{13-16}$

\section{Statistical Analysis}

Patient characteristics were described using medians and interquartile ranges (IQRs) for continuous variables and frequencies (percentages) for categorical variables. Effect sizes were calculated using Cohen's $d$ or Cramer's $V$. To account for patient-level correlation, we estimated beta coefficients $(\beta)$ and $95 \%$ confidence intervals evaluating the difference between patient cost responsibility by receipt of guideline-discordant treatment using linear mixed-effect models. Overall and healthcare servicestratified models were adjusted for patient demographic and clinical characteristics, including index diagnosis year, age at index diagnosis, race, MBC type, HR status, HER2 status, NCI comorbidity index score, Medicare/ Medicaid dual-eligibility status, and census track education level. Sensitivity analyses were conducted for overall discordance and by category of guideline discordance using generalized linear models with a gamma distribution and log-link. Analyses were performed using SAS 9.4 (SAS Institute, Inc).

\section{Results}

Of 3,709 patients with MBC (median age, 69 years [IQR, 64-77]), 17.6\% $(n=651)$ received treatment discordant with NCCN Guidelines for Breast Cancer. Demographic and clinical characteristics for the study sample are shown in Table 1. Compared with patients receiving guideline-concordant care, those receiving discordant treatment were younger and were more often Medicare/ Medicaid dual-eligible, HR-negative, and HER2-positive. Median patient cost responsibility in the year after index diagnosis was significantly higher for guideline-discordant versus -concordant care $(\$ 7,421$ [IQR $\$ 4,359-\$ 12,983$ ] vs $\$ 5,171$ [IQR \$3,006-\$8,483]; $P<.001$ ).

After adjustments for patient demographic and clinical characteristics, guideline-discordant care was associated with significantly higher patient cost responsibility in the first year following index diagnosis $(\beta=\$ 1,841 ; 95 \% \mathrm{CI}$, $\$ 1,280-\$ 2,401)$ compared with guideline-concordant treatment. In addition, significant healthcare servicestratified patient cost responsibility differences were found for outpatient ( $\beta=\$ 1,288$; 95\% CI, $\$ 800-\$ 1,776$ ), physician visit/carrier ( $\beta=\$ 326$; 95\% CI, $\$ 204-\$ 449$ ), and Medicare Part D costs ( $\beta=\$ 95 ; 95 \%$ CI, $\$ 1-\$ 189)$ between discordant and concordant treatment (Figure 2).

Figure 3 presents results from the adjusted models evaluating the association of patient cost responsibility with categories of guideline-discordant treatments. Patients receiving nonapproved bevacizumab $(n=58 / 651$; $9 \%$ ) had the highest cost responsibility when compared with those receiving guideline-concordant treatment ( $\beta=\$ 3,330 ; 95 \%$ CI, $\$ 1,711-\$ 4,948)$. Patients receiving HER2-targeted therapy without chemotherapy ( $n=247 / 651$; $38 \%$ ) also had significantly higher costs than those receiving concordant treatment $(\beta=\$ 2,078 ; 95 \% \mathrm{CI}$, $\$ 1,252-\$ 2,904)$, whereas those receiving therapy mismatched with their HR or HER2 status ( $n=46 / 651 ; 7 \%$ ) had significantly lower costs than those receiving concordant treatment ( $\beta=-\$ 3,912 ; 95 \% \mathrm{CI},-\$ 5,796$ to $-\$ 2,027)$. Costs for patients receiving adjuvant regimens in the metastatic setting $(\mathrm{n}=73 / 651 ; 11 \%)$ did not significantly differ from costs for those receiving guideline-concordant treatments $(\beta=\$ 1,196 ; 95 \% \mathrm{CI},-\$ 260$ to $\$ 2,651)$. Sensitivity analyses using generalized linear models showed similar results by overall and category of guideline discordance.

\section{Discussion}

This is the first study to show that receipt of guidelinediscordant treatment is associated with significantly higher patient-specific cost responsibility for patients with MBC. This significant cost increase was seen for 
Table 1. Patient Characteristics $(N=3,709)$

\begin{tabular}{|c|c|c|c|}
\hline & $\begin{array}{c}\text { Guideline-Discordant } \\
\mathbf{n}(\%)\end{array}$ & $\begin{array}{c}\text { Guideline-Concordant } \\
\text { n (\%) }\end{array}$ & Effect Size \\
\hline Total, N & 651 & 3,058 & \\
\hline Race & & & .03 \\
\hline White & $487(74.8)$ & $2,392(78.2)$ & \\
\hline MBC type & & & .02 \\
\hline De novo & $136(20.9)$ & $578(18.9)$ & \\
\hline Secondary metastatic disease & $515(79.1)$ & $2,480(81.1)$ & \\
\hline Hormone receptor status ${ }^{a}$ & & & .29 \\
\hline HER2 status & & & .18 \\
\hline Positive & $64(9.8)$ & $51(1.7)$ & \\
\hline Negative & $38(5.8)$ & $338(11.1)$ & \\
\hline Unknown & $549(84.3)$ & $2,669(87.3)$ & \\
\hline $\mathrm{NCl}$ comorbidity index score, median (IOR) & $0(0-1)$ & $0(0-1)$ & .06 \\
\hline Medicare/Medicaid dual-eligibility status & & & .04 \\
\hline Never dual-eligible & $362(55.6)$ & $1,869(61.1)$ & \\
\hline Dual-eligible & $289(44.4)$ & $1,189(38.9)$ & \\
\hline Census tract education level, ${ }^{\mathrm{b}}$ median (IQR) & $82(70-90)$ & $83(71-91)$ & .10 \\
\hline
\end{tabular}

Abbreviations: IQR, interquartile range; $M B C$, metastatic breast cancer.

aEstrogen/Progesterone receptor.

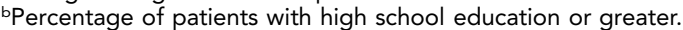

both overall and healthcare service-stratified care costs, including outpatient, physician visit/carrier, and Medicare Part D costs. This study adds to previously published studies about guideline discordance focused on costs to payers, with most finding higher payer costs associated with treatment that is discordant with NCCN Guidelines. For example, when considering costs from a societal perspective, one study reported that an estimated \$2.5 billion was spent in 2010 for 10 common chemotherapies that were used in discordance with NCCN recommendations. ${ }^{17}$ Similarly, in a SEER-Medicare analysis of almost 10,000 patients with ovarian cancer, Urban et al ${ }^{18}$ found $\$ 7,500$ higher Medicare spending for patients receiving guideline-discordant chemotherapy and surgery versus for concordant treatment in the first year after diagnosis. Although these and other studies have shown associations between guideline discordance and increased payer costs, patients themselves are largely unaware of and indifferent to payer or societal care costs outside of their immediate responsibility. ${ }^{19-21}$ Therefore, considering costs from the patient perspective has considerable clinical relevance in individual-level treatment decisions.
Previous research has found differences in mortality by category of guideline discordance, despite a lack of difference when comparing concordance versus discordance of overall treatment. ${ }^{4,5}$ Our study adds that the category of guideline discordance also influences patient cost responsibility. Importantly, the category with the highest patient cost responsibility-nonapproved bevacizumab-was associated with both an increased patient cost responsibility of almost $\$ 12,000$ in the first year after diagnosis and a $40 \%$ increased hazard of mortality (hazard ratio, 1.40; 95\% CI, 1.13-1.74). ${ }^{5}$ However, this category did not include patients who received paclitaxel with bevacizumab, because this combination was included in the NCCN Guidelines during our study period based on its accelerated approval for use in MBC in 2008. Ultimately, bevacizumab was found to pose lifethreatening adverse effects with minimal benefit to patients with MBC, and its indication was removed in late 2010. ${ }^{22}$ Although the FDA does not consider cost-effectiveness during drug approval, one study found a cost to payers of $\$ 745,000$ per quality-adjusted life-year in patients with $\mathrm{MBC}$ receiving combination bevacizumab/paclitaxel 


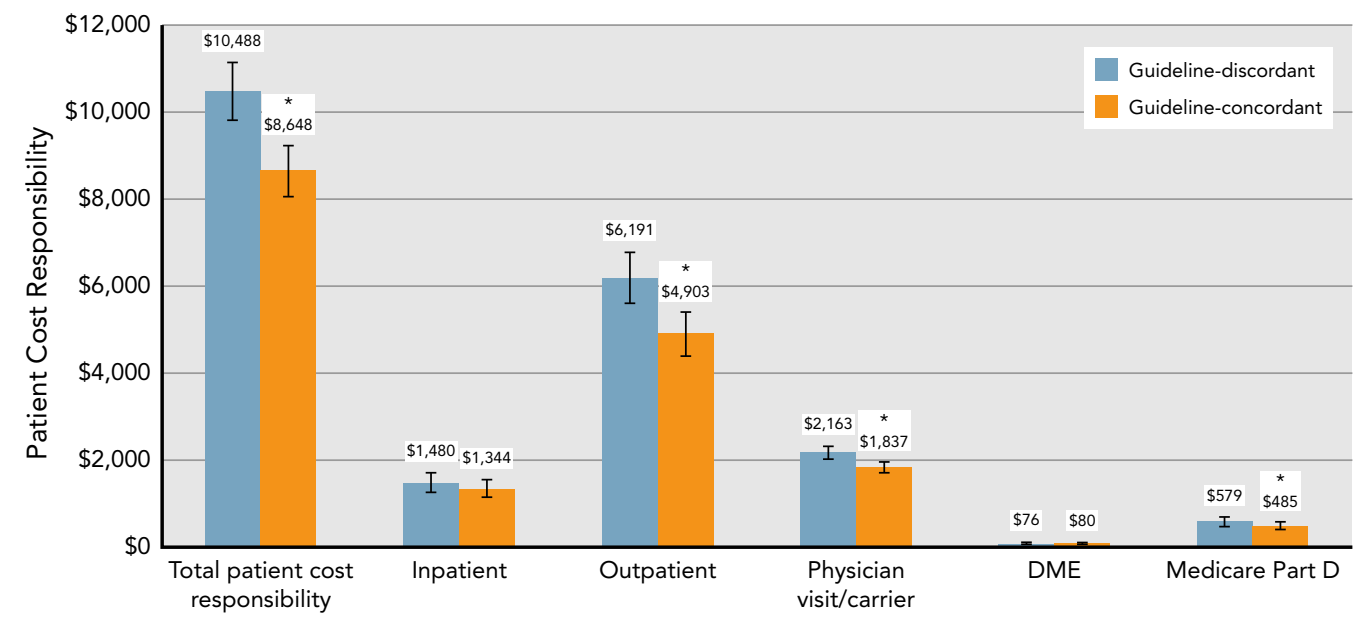

Figure 2. Model-adjusted patient cost responsibility overall and stratified by healthcare services in the year after index MBC diagnosis (guideline-discordant, $n=651$; guideline-concordant, $n=3,058$ ). Models were adjusted for index diagnosis year, age at index diagnosis, race, MBC type, hormone receptor status, HER2 status, $\mathrm{NCl}$ comorbidity index score, Medicare/Medicaid dual-eligibility status, and census track education level.

Abbreviations: DME, durable medical equipment; MBC, metastatic breast cancer.

*Significant difference from guideline-concordant status $(P<.05)$.

compared with paclitaxel alone. ${ }^{23}$ Our study shows that this extreme cost is not only restricted to payers but also passed down to patients. This effect may be exacerbated in the future as payers implement clinical pathway programs, potentially resulting in claim denial due to receipt of guideline-discordant treatment. This example represents a cautionary tale for physicians who add novel agents without proven benefit to treatment regimens, potentially incurring substantial patient costs, and also highlights the fact that guidelines may change over time. In formulating novel drugs or treatment guidelines, an emphasis on more patient-centered outcomes, such as decreased financial burden, prolonged overall survival, and quality of life, can be as valuable to patients as progression-free survival.

The lower cost responsibility seen for patients receiving therapy mismatched with HR/HER2 status was predominantly due to the use of oral hormone therapy in older patients with HR-negative MBC. We suspect that some physicians may have elected to prescribe hormone

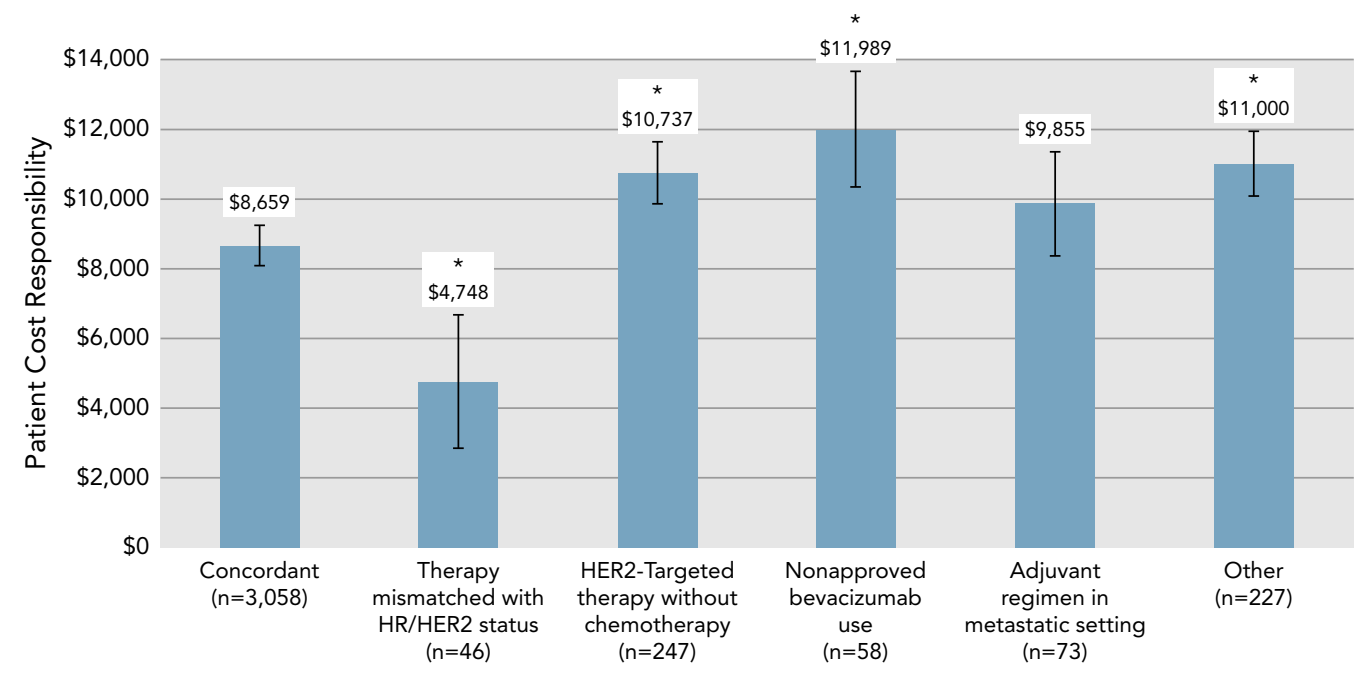

Figure 3. Model-adjusted overall patient cost responsibility by type of NCCN Guideline discordance in the year after index MBC diagnosis. Models were adjusted for index diagnosis year, age at index diagnosis, race, $\mathrm{MBC}$ type, hormone receptor status, $\mathrm{HER} 2$ status, $\mathrm{NCl}$ comorbidity index score, Medicare/Medicaid dual-eligibility status, and census track education level.

Abbreviations: HR, hormone receptor; MBC, metastatic breast cancer.

*Significant difference from guideline-concordant status $(P<.05)$. 
therapy for patients with HR-negative MBC deemed ineligible for chemotherapy. Although often well-tolerated, hormone therapy has adverse effects, such as hot flashes, weight gain, insomnia, and joint aches, with one study reporting that $46 \%$ of patients with ER-positive breast cancer took medication to control symptoms. ${ }^{24}$ Ultimately, no treatment may be a better choice than a guideline-discordant treatment associated with mild but persistent and bothersome adverse effects.

Conversely, higher cost responsibility was seen for patients receiving HER2-targeted therapy without chemotherapy. We believe this is due to HER2-targeted therapies being more expensive than hormonal therapies, and suspect that patients who may not otherwise tolerate chemotherapy are given HER2-targeted therapies without chemotherapy due to increased disease severity, adverse treatment adverse effects, or decreased functional status. These issues may result in increased patient spending on more frequent clinic visits, supportive medications to reduce treatment adverse effects, or supplies to address decreased functional status, such as walkers. More research is needed to determine how to effectively manage older patients with MBC experiencing treatment-related adverse effects, especially because a clinical benefit has been suggested for guideline-discordant single-agent trastuzumab as first-line monotherapy. ${ }^{25}$

Little guidance exists on the optimal treatment strategy for older adults with MBC. Randomized clinical trials, which largely guide MBC treatment evidence, often exclude patients with advanced age due to eligibility criteria with restrictions on comorbidities and functional status. ${ }^{26}$ In studies of older adults with early-stage breast cancer, undertreatment with adjuvant capecitabine alone doubled the risk of death compared with standard chemotherapy, ${ }^{27}$ and overtreatment with lumpectomy plus tamoxifen and radiation therapy has shown no benefit compared with lumpectomy plus adjuvant therapy with tamoxifen alone. ${ }^{28}$ In our study of patients with MBC, with $74 \%$ of the patient sample aged $\geq 65$ years and $31 \%$ aged $\geq 75$ years, deviations from the NCCN Guidelines were noted in terms of both overtreatment, such as adjuvant regimens given in the metastatic setting, and undertreatment, such as HER2-targeted therapy given without chemotherapy, both of which resulted in higher patient costs compared with receipt of guideline-concordant care. These higher costs could be particularly impactful for older adults, who could incur the increased burden of out-ofpocket healthcare costs even with supplemental insurance, because most encounter a large decrease in annual income after retirement. Therefore, incorporating a geriatric assessment and considering patient preferences surrounding therapy costs are recommended to optimize treatment decision-making in older adults based on functional status, comorbidity, medication use, nutritional status, social status, cognition, and psychological concerns rather than chronologic age alone. ${ }^{29,30}$

These findings underscore the potential for treating oncologists to influence patient cost responsibility based on treatment choice. Evidence has suggested that few oncologists are comfortable discussing costs with their patients, although most individuals with cancer are interested in discussion about out-of-pocket costs. ${ }^{19,21,31,32}$ This type of cost information is difficult to consider and often challenging to relay to patients due to continual changes in insurance coverage, drug costs, and healthcare policy. Despite this concern, oncologists believe that patient financial burden is an issue to be addressed. In a national survey of medical oncologists, $84 \%$ responded that patient costs would influence treatment recommendations. ${ }^{33}$ Kelly et $\mathrm{al}^{34}$ found that $83 \%$ of oncologists at an academic institution wished cost information was included in the NCCN Guidelines, potentially creating greater ease in patient-physician cost conversations. Although NCCN Evidence Blocks provide an estimate of the total treatment cost as a measure of value, a direct calculation of patient out-of-pocket costs is not included. ${ }^{35}$ Tools such as the ASCO Value Framework could be useful to guide both oncologists and patients with $\mathrm{MBC}$ in treatment decision-making when paired with NCCN Guidelines, because the Framework considers patient copay in its net health benefit calculation of a particular cancer treatment regimen. ${ }^{36,37}$ Thus, our work is critical, as we show that deviation from guidelines may influence patient healthcare expenses and that oncologists can play an important role in addressing patient financial burden.

Our results should be considered in the context of several limitations. Claims analysis is limited by the inability to identify who paid for patient-specific costs. ${ }^{38}$ Although we could not measure exact out-of-pocket costs for included patients, the cost estimates represent the direct medical costs for those without supplemental insurance and show the mirroring of patient and payer costs. Furthermore, the estimates herein likely underestimate the true patient out-of-pocket costs of cancer-related care, which could include indirect costs such as transportation, lodging, cosmetic or therapeutic items, mental health services, caregiving/childcare, and lost wages due to absence from work..$^{39,40}$ Due to the set inclusion criteria, results may not be generalizable to patients with MBC who survive $<1$ year from diagnosis. Likewise, patient cost responsibility was not considered outside of 1 year postdiagnosis. We also did not consider the effect of catastrophic coverage on patient cost responsibility. Unmeasured confounders influencing patient costs may still exist because of the inability to capture variables using SEER-Medicare data, such as illness severity, functional status, or frailty. Claims for secondary malignancy also 
vary in completeness and accuracy. Finally, a completely causal relationship between guideline discordance and patient cost responsibility cannot be established based on the current data or study design.

\section{Conclusions}

Our results showed that patient cost responsibility was nearly $\$ 2,000$ higher for the approximately $18 \%$ of patients with MBC receiving NCCN Guideline-discordant versus - concordant care. These findings have important implications for training clinicians in terms of the importance of guideline-concordant care and the potential impact on patient cost responsibility. Our work can inform efforts to improve patient-clinician communication about the value of guideline-based care and the potential implications of financial burden associated with cancer treatments. As the costs of cancer care continue to increase and physicians shift to more value-based healthcare delivery systems, ongoing research should build on these findings to develop effective solutions targeting standardized treatments to avoid potentially unnecessary financial toxicity.

\section{Acknowledgments}

This study used the linked SEER-Medicare database. The interpretation and reporting of these data are the sole responsibility of the authors. The authors acknowledge the efforts of the NCI; the Office of Research, Development, and Information, Centers for Medicare \& Medicaid Services; Information Management Services, Inc.; and the SEER program tumor registries in the creation of the SEER-Medicare database.

\section{Submitted January 8, 2019; accepted for publication April 30, 2019}

Previous presentation: This work was presented as an oral presentation at the Society for Medical Decision Making 40th Annual North American Meeting October 13-17, 2018; Montreal, Quebec, Canada.

Author contributions: Study concept/design: Williams, Rocque. Provision of study material or patients: Rocque. Data collection/assembly: Williams, Azuero. Data analysis and interpretation: Williams, Azuero, Kenzik, Rocque. Manuscript writing: All authors. Final approval of manuscript: All authors.

Disclosures: Dr. Rocque has disclosed that she has received grant/research support from CareVive, Genentech, and Pfizer, and consulting fees/honoraria from Pfizer and Roche. All remaining authors have not received any financial consideration from any person or organization to support the preparation, analysis, results, or discussion of this article.

Correspondence: Courtney P. Williams, MPH, Division of Hematology and Oncology, University of Alabama at Birmingham, WTI 240, 1720 2nd Avenue South, Birmingham, AL 35294. Email: courtneyphillips@uabmc.edu

\section{References}

1. Newcomer LN, Malin JL. Payer view of high-quality clinical pathways for cancer. J Oncol Pract 2017;13:148-150.

2. Gradishar WJ, Anderson BO, Abraham J, et al. NCCN Clinical Practice Guidelines in Oncology: Breast Cancer. Version 2.2019. Accessed July 31, 2019. To view the most recent version, visit NCCN.org

3. Gradishar WJ, Anderson BO, Balassanian R, et al. NCCN Guidelines Insights: Breast Cancer. Version 1.2016. J Natl Compr Canc Netw 2015; 13:1475-1485.

4. Rocque GB, Williams CP, Jackson BE, et al. Impact of nonconcordance with NCCN Guidelines on resource utilization, cost, and mortality in de novo metastatic breast cancer. J Natl Compr Canc Netw 2018;16:1084-1091.

5. Rocque GB, Williams CP, Kenzik KM, et al. Concordance with NCCN treatment guidelines: relations with health care utilization, cost, and mortality in breast cancer patients with secondary metastasis. Cancer 2018;124:4231-4240

6. Zafar SY, Abernethy AP. Financial toxicity, part I: a new name for a growing problem. Oncology (Williston Park) 2013;27:80-149.

7. Kaisaeng N, Harpe SE, Carroll NV. Out-of-pocket costs and oral cance medication discontinuation in the elderly. J Manag Care Spec Pharm 2014;20:669-675

8. Zafar SY, McNeil RB, Thomas CM, et al. Population-based assessment of cancer survivors' financial burden and quality of life: a prospective cohort study. J Oncol Pract 2015;11:145-150.

9. Warren JL, Klabunde CN, Schrag D, et al. Overview of the SEER-Medicare data: content, research applications, and generalizability to the United States elderly population. Med Care 2002;40(Suppl 8):IV-3-18.

10. Danese MD, Lindquist K, Doan J, et al. Effect of central nervous system metastases on treatment discontinuation and survival in older women receiving trastuzumab for metastatic breast cancer. J Cancer Epidemiol 2012;2012:819210.

11. Stokes ME, Thompson D, Montoya EL, et al. Ten-year survival and cost following breast cancer recurrence: estimates from SEER-Medicare data. Value Health 2008;11:213-220.

12. Frank B. Definitions of "cost' in Medicare utilization files. Research Data Assistance Center. Available at http://resdac.umn.edu/sites/resdac.umn. edu/files/Definitions\%20of\%20\%27Cost\%27\%20in\%20Medicare\%20Utilization\%20Files\%20(Slides).pdf. Accessed May 21, 2019.

13. Klabunde CN, Potosky AL, Legler JM, et al. Development of a comorbidity index using physician claims data. J Clin Epidemiol 2000;53: 1258-1267.
14. Charlson ME, Pompei $\mathrm{P}$, Ales $\mathrm{KL}$, et al. A new method of classifying prognostic comorbidity in longitudinal studies: development and validation. J Chronic Dis 1987;40:373-383.

15. Deyo RA, Cherkin DC, Ciol MA. Adapting a clinical comorbidity index for use with ICD-9-CM administrative databases. J Clin Epidemiol 1992;45: 613-619.

16. Romano PS, Roos LL, Jollis JG. Adapting a clinical comorbidity index for use with ICD-9-CM administrative data: differing perspectives. J Clin Epidemiol 1993;46:1075-1079; discussion 1081-1090.

17. Conti RM, Bernstein AC, Villaflor VM, et al. Prevalence of off-label use and spending in 2010 among patent-protected chemotherapies in a populationbased cohort of medical oncologists. J Clin Oncol 2013;31:1134-1139.

18. Urban RR, He H, Alfonso-Cristancho $\mathrm{R}$, et al. The cost of initial care for Medicare patients with advanced ovarian cancer. J Natl Compr Canc Netw 2016;14:429-437.

19. Bullock AJ, Hofstatter EW, Yushak ML, et al. Understanding patients' attitudes toward communication about the cost of cancer care. J Oncol Pract 2012;8:e50-58.

20. Meisenberg BR, Varner A, Ellis E, et al. Patient attitudes regarding the cost of illness in cancer care. Oncologist 2015;20:1199-1204.

21. Irwin B, Kimmick G, Altomare I, et al. Patient experience and attitudes toward addressing the cost of breast cancer care. Oncologist 2014;19: 1135-1140

22. Montero AJ, Escobar M, Lopes G, et al. Bevacizumab in the treatment of metastatic breast cancer: friend or foe? Curr Oncol Rep 2012; 14:1-11.

23. Montero AJ, Avancha K, Glück S, et al. A cost-benefit analysis of bevacizumab in combination with paclitaxel in the first-line treatment of patients with metastatic breast cancer. Breast Cancer Res Treat 2012;132: 747-751.

24. Garreau JR, Delamelena T, Walts D, et al. Side effects of aromatase inhibitors versus tamoxifen: the patients' perspective. Am J Surg 2006;192:496-498.

25. Vogel CL, Cobleigh MA, Tripathy D, et al. First-line Herceptin monotherapy in metastatic breast cancer. Oncology 2001;61(Suppl 2):37-42.

26. Townsley CA, Selby R, Siu LL. Systematic review of barriers to the recruitment of older patients with cancer onto clinical trials. J Clin Oncol 2005;23:3112-3124.

27. Muss HB, Berry DA, Cirrincione CT, et al. Adjuvant chemotherapy in older women with early-stage breast cancer. N Engl J Med 2009;360: 2055-2065. 
28. Hughes KS, Schnaper LA, Berry D, et al. Lumpectomy plus tamoxifen with or without irradiation in women 70 years of age or older with early breast cancer. N Engl J Med 2004;351:971-977.

29. Jolly TA, Williams GR, Bushan S, et al. Adjuvant treatment of older women with invasive breast cancer. Womens Health (Lond) 2016;12:129-146.

30. Jolly $T$, Williams GR, Jones $E$, et al. Treatment of metastatic breast cancer in women aged 65 years and older. Womens Health (Lond) 2012;8:455-471.

31. Kelly RJ, Forde PM, Elnahal SM, et al. Patients and physicians can discuss costs of cancer treatment in the clinic. J Oncol Pract 2015;11: 308-312.

32. Henrikson NB, Tuzzio L, Loggers ET, et al. Patient and oncologist discussions about cancer care costs. Support Care Cancer 2014;22:961-967.

33. Neumann PJ, Palmer JA, Nadler E, et al. Cancer therapy costs influence treatment: a national survey of oncologists. Health Aff (Millwood) 2010;29: 196-202.

34. Kelly RJ, Forde PM, Bagheri A, et al. Measuring the impact of chemotherapy cost discussions between patients and providers at the time of prescribing [abstract]. J Clin Oncol 2013;31(Suppl):Abstract 257.
35. Chandra A, Shafrin J, Dhawan R. Utility of cancer value frameworks for patients, payers, and physicians. JAMA 2016;315:2069-2070.

36. Schnipper LE, Davidson NE, Wollins DS, et al. American Society of Clinical Oncology statement: a conceptual framework to assess the value of cancer treatment options. J Clin Oncol 2015;33:2563-2577.

37. Schnipper LE, Davidson NE, Wollins DS, et al. Updating the American Society of Clinical Oncology value framework: revisions and reflections in response to comments received. J Clin Oncol 2016: 34:2925-2934.

38. Erten $\mathrm{MZ}$, Davidoff AJ, Zuckerman IH, et al. The effect of supplemental medical and prescription drug coverage on health care spending for Medicare beneficiaries with cancer. Value Health 2014; 17:15-21.

39. Wan $Y$, Gao X, Mehta $S$, et al. Indirect costs associated with metastatic breast cancer. J Med Econ 2013;16:1169-1178.

40. Arozullah AM, Calhoun EA, Wolf $M$, et al. The financial burden of cancer: estimates from a study of insured women with breast cancer. J Support Oncol 2004:2:271-278.

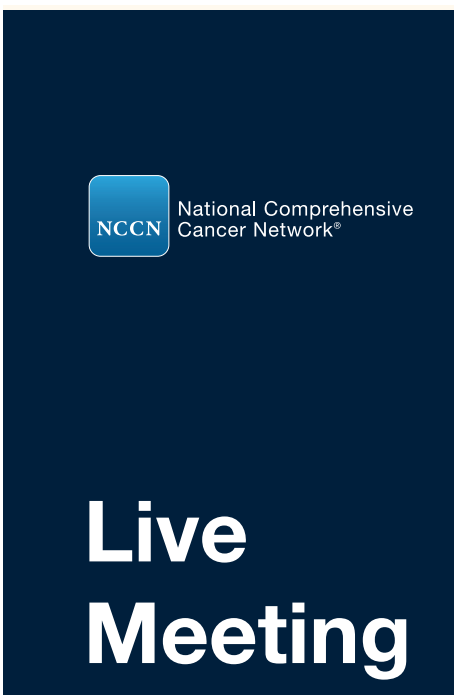

REGISTER NOW
NCCN PATIENT ADVOCACY SUMMIT:

\section{Delivering Value for Patients Across the Oncology Ecosystem}

\author{
Wednesday, December 11, 2019 \\ The National Press Club \\ Washington, DC
}

Supported by Adaptive Biotechnologies; AmerisourceBergen; an educational donation provided by Amgen; Apobiologix; Astellas; AstraZeneca through its NCCN Corporate Council Membership; Celgene Corporation; Coherus BioSciences, Inc.; Curium; Eisai Inc.; EMD Serono; Ferring Pharmaceuticals; Foundation Medicine, Inc.; Genentech; HELSINN; Heron; Incyte Corporation; Janssen Oncology, Pharmaceutical Companies of Johnson \& Johnson; Kite, a Gilead Company; an independent educational grant from Merck \& Co.., Inc.; Novartis Oncology; Regeneron Pharmaceuticals Inc; Sanofi; Servier; Sirtex; Taiho Oncology; Takeda; TESARO, A GSK Company; and Verastem Oncology. Sponsored by Boehringer Ingelheim Pharmaceuticals, Inc., Bristol-Myers Squibb, Dendreon Pharmaceuticals LLC. This activity was made possible by

\section{Visit NCCN.org/policy to register.}

